\title{
OBLIGACIONES DE LA MATERIALIDAD EN OPERACIONES DE LOS CONTRIBUYENTES
}

\section{MATERIALITY OBLIGATIONS IN TAXPAYERS OPERATIONS THE NECESITY OF BITCOIN'S ACCOUNTING, LEGAL AND FISCAL REGULATION IN MEXICO, A TOPICAL ISSUE}

Lic. Samuel Zeniff Cornejo Martínez ${ }^{\mathrm{a}}$

Dra. María del Carmen García García ${ }^{\text {b }}$

Lic. Esther Chávez Cruz ${ }^{c}$

Lic. Ricardo Colorado Colorado ${ }^{d}$

\author{
${ }^{a}$ Universidad Veracruzana \\ Instituto de Investigaciones en Contaduría, zeniffcornejo@ hotmail.com \\ ${ }^{\mathrm{b}}$ Universidad Veracruzana \\ Instituto de Investigaciones en Contaduría, cgarcia@uv.mx \\ ${ }^{\mathrm{c}}$ Universidad Veracruzana \\ Instituto de Investigaciones en Contaduría, chavez_cruz@hotmail.com \\ ${ }^{\mathrm{d}}$ Universidad Veracruzana \\ Instituto de Investigaciones en Contaduría, ricardo.coloradoc2@gmail.com
}

Fecha de aceptación: 25 de junio de 2020

\section{RESUMEN}

Resultado de la globalización que se experimenta hoy en día son los cambios que surgen en materia tributaria en la adecuación de las normas con el uso de Tecnologías de Información y Comunicaciones (TIC's), y los requisitos de los Comprobantes Fiscales Digitales por Internet (CFDI) porque la autoridad tiene más información sobre el contribuyente al tener conocimiento de primera mano de las operaciones realizadas durante un cierto período, ya que la propia autoridad autoriza su emisión. Una vez que se han emitido los comprobantes, es el derecho del contribuyente deducir el gasto y acreditar el impuesto correspondiente. Sin embargo, la autoridad fiscal considera necesario demostrar la materialidad para verificar dicha transacción. Este trabajo revela la importancia de tener contratos en la venta comercial, la forma de probar el fondo de la transacción y su efecto fiscal. El contenido destaca la relación entre el comprador y el vendedor y como un hecho comercial del que surgen las obligaciones 
tributarias a las que los contribuyentes están sujetos a cumplimiento. En una revisión de la información localizada y la experiencia con las verificaciones practicadas por la autoridad fiscal, se formen juicios sobre las facultades de comprobación.

PAlabRAS CLAVE: Materialidad; Deducibilidad; Contribuyentes; Certeza; Transacción.

\begin{abstract}
Result of the globalization that is experienced today are the changes that arise in tax matters in the adequacy of the rules with the use of information and communication technologies (TIC), and the requirements of digital internet tax receipts (CFDI) because the authority has more information on the taxpayer by having first-hand knowledge of the operations carried out during a certain period, since the authority itself authorizes its issuance. Once the vouchers have been issued, it is the taxpayer's right to deduct the expense and credit the corresponding tax. However, the tax authority considers it necessary to demonstrate materiality to verify said transaction. This work reveal the importance of having contracts in commercial sale, the way in which to prove the substance of the transaction and its tax effect. The content highlights the relationship between buyer and seller and as a commercial fact from which tax obligations to which taxpayers are subject for compliance arise. In a review of localized information and experience with verifications practiced by the tax authority, form judgments about the powers of verification.
\end{abstract}

KEYWORDS: Transaction Materiality; Deducibility; Taxpayers; Certainty; Transaction

\title{
I. INTRODUCCIÓN
}

Las empresas que se encuentran en el mercado comercial, tratando de mantenerse en el competitivo mundo globalizado, como parte de sus operaciones, realizan compras de bienes que posteriormente ponen a la venta.

Con ello obtienen ingresos y egresos. Lo primero es por las ventas y lo segundo es por las compras. Desde el momento en que realizan operaciones comerciales se registran en el padrón de contribuyentes, que el servicio de Administración Tributaria (SAT) utiliza para generar las 
obligaciones fiscales a las cuales se encuentran sujetos, y por consiguiente deben calcular su impuesto por las operaciones formalizadas con terceros.

Aquí es donde se ubica el problema de análisis, al determinar sus impuestos, los documentos que reciben o emiten son su soporte de demostrar que se ha recibido ingreso o realizando gastos, llamados Comprobantes Fiscales Digitales por Internet (CFDI) donde los comprobantes que se utilizan para deducir han ido perdiendo la certeza jurídica que habían mantenido por años. El propósito del artículo es demostrar que la certeza jurídica juega un papel importante en la materialidad de las operaciones de un contribuyente en su ejercicio del derecho de deducir el gasto y acreditar el impuesto

La metodología empleada en este trabajo lo ubica como una investigación de corte cualitativo, de acuerdo con Bernal (2010); parte de un hecho específico que están impactados los contribuyentes que solicitan una devolución, que en el objeto de estudio representa un $40 \%$ de los contribuyentes registrados. Para argumentar se utiliza el método deductivo partiendo de la generalidad de la norma a la aplicación particular y los efectos en el ejercicio de facultades de comprobación. Es una investigación descriptiva más no limitativa que implica una situación recurrente en los contribuyentes en condiciones similares.

Se parte de las normas fiscales que indican los derechos y obligaciones de los contribuyentes, los cuales al realizar operaciones para generar sus ingresos están causando un impuesto a pagar o un impuesto a favor; este segundo da derecho a solicitar su devolución. Es aquí donde parte el problema objeto de estudio cuando al realizar la solicitud de devolución la autoridad fiscal realiza la verificación de la documentación y exige la demostración de la materialidad de las operaciones, de lo contrario podría variar la base gravable y el monto a devolver.

El objetivo del presente artículo es analizar y demostrar la importancia de la certeza jurídica de la deducibilidad de los CFDI y sus efectos en la base gravable determinada por el contribuyente. 


\section{MARCO TEÓRICO}

\section{1 Régimen tributario}

Los contribuyentes motivo de este análisis son aquellos que para la norma se encuentran clasificados por sus características, forma de integración, por tipo de sociedad, tipo de actividad empresarial, monto de ingresos, entre otras.

Para la funcionalidad de la empresa es necesario de equipar con lo indispensable para la realización de su actividad económica preponderante, como lo son las inversiones en maquinaria, equipo, edificio, equipo de cómputo, insumos, etc.

Para que todos los registros tanto de ingresos o egresos tengan una coherencia entre si es necesario que se cumpla con los requisitos que establece el Artículo 28 del Código Fiscal de la Federación (CFF) de llevar contabilidad integrada por: "los libros, sistemas y registros contables, papeles de trabajo, estados de cuenta, cuentas especiales, libros y registros sociales, control de inventarios y método de valuación, discos y cintas o cualquier otro medio..." (Código Fiscal de la Federación, 2019, pág. 66).

Al cumplir con el requisito de llevar control en los registros de los movimientos económicos del contribuyente tanto de las ventas como de las compras, se debe cumplir con lo establecido en la Ley del Impuesto Sobre la Renta (LISR) en su Artículo 27 dando que con las deducciones se tiene el efecto legal de disminuir ingresos, a este concepto se le conoce comúnmente como gastos.

"Los contribuyentes se ven inmersos en actividades mercantiles por cada acción de compraventa que realicen, ...donde todas las adquisiciones, enajenaciones verificados con propósitos de especulación comercial son consideradas actos de comercio", Artículo 75. (Código de Comercio, 2018).

En los efectos fiscales del trato mercantil, se debe distinguir el tipo de régimen tributario que se opta, para encuadrar en las obligaciones fiscales y jurídicas del contribuyente.

La Ley del Impuesto Sobre la Renta (LISR), señala diferentes regímenes de tributar siendo: 
- Persona moral del título II

- Persona moral del título III

- Personas físicas con actividad empresarial del título IV capítulo II sección I

- Personas físicas con actividad empresarial del título IV capítulo II sección II

- Personas físicas con actividad empresarial del título IV capítulo III, entre otros

Una vez definido el régimen de tributación es necesario dar cumplimiento a dicha ley en cuanto a la deducibilidad y acumulación de ingresos por las operaciones mercantiles del contribuyente que se trate.

La figura 1 muestra información del Régimen Tributario de estudio de esta investigación.

\section{Figura 1}

Padrón oficial del SAT por régimen fiscal

\section{Régimen de las personas físicas con actividades empresariales y profesionales}

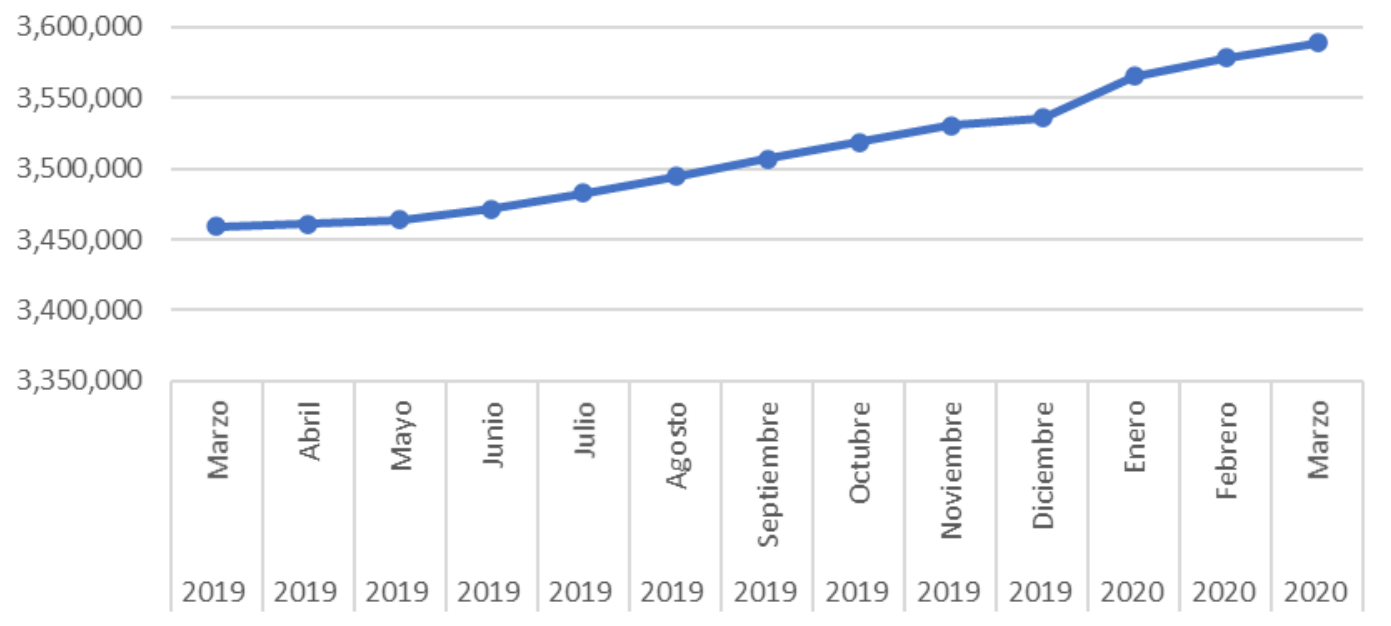

Fuente: Elaboración propia con datos del SAT 2019

Puede observarse en la información estadística que proporciona el SAT, como el número de personas físicas con actividad empresarial ha ido incrementando por lo cual se deduce que la misma globalización está orillando a realizar la inscripción para poder concretar la enajenación de sus productos.

Por lo tanto, para determinar la base gravable en la determinación de los impuestos a este tipo 
de régimen tributario se realizará la disminución de "la totalidad de los ingresos comprendido desde el inicio del ejercicio y hasta el último día del mes que corresponde el pago, las deducciones autorizadas en esta sección correspondientes al mismo periodo", como lo establece el Artículo 106 LISR (Ley del Impuesto Sobre la Renta, 2019).

Entonces lo contribuyentes deben tener mucho cuidado de que sus gastos cumplan con los requisitos establecidos en ley para su deducción y así no se vean perjudicados en la base gravable y el pago del impuesto será el justo.

\section{2 Deducciones}

Los gastos tienen características específicas que deben cumplir, según lo estipula la LISR como lo establece su Artículo 105 (Ley del Impuesto Sobre la Renta, 2019), deben de ser:

- Estrictamente indispensables para llevar a cabo las actividades económicas del contribuyente

- Que hayan sido efectivamente erogadas en el ejercicio en que se trate (pagados en efectivo, transferencia bancaria, cheque, entre otros)

- Que se resten una sola vez, aun cuando estén relacionadas con la obtención de diversos ingresos.

Puede observarse que no hace alusión a un requisito esencial en el uso de los CFDI para comprobar la realización de actividades económicas de una manera legítima.

Los contribuyentes tienen como obligación "expedir los comprobantes fiscales por los actos o actividades que realicen, por los ingresos que se perciban o por las retenciones de contribuciones que efectúen”, Artículo 29 CFF (Código Fiscal de la Federación, 2019, pág. $68)$.

Por lo que al ser un ingreso del contribuyente que enajena, es obligación realizar la expedición del comprobante que ampara dicha operación, es así como el SAT regula al contribuyente por medio de la facturación electrónica. Mismo caso se presenta cuando se realizan los gastos por los insumos, invirtiendo la posición del contribuyente que ahora realiza la acción de 
adquisición y por ello igualmente debe recibir un CFDI.

Cabe mencionar que la facturación electrónica ha ido evolucionando, tomando en consideración las TIC's lo que privilegia de información oportuna, real y vigente de ingresos y gastos que generan los contribuyentes, a las autoridades fiscales SAT al validar la emisión de los CFDI mencionado en el Artículo 105 de LISR y éste es de aplicación estricta.

Se debe conocer que el CFF señala los requisitos que deben cumplir los CFDI, para que la autoridad fiscal de el visto bueno de cumplir las disposiciones fiscales para la deducción, conforme al Artículo 29-A y son los siguientes:

- Contar con RFC del emisor

- Contar con número de folio fiscal y sello digital

- Código postal del lugar donde se expidió el CFDI

- Unidad de medida, cantidad y clase de bienes que se enajenen

- Valor unitario del producto o servicio

- Impuesto desglosado y por separado del valor unitario

- Valor total del bien

- Número y fecha del documento aduanero en casi de importación

Es importante conocer la legislación que regula los temas tributarios en el país porque al tener un desconocimiento de este tema en particular impacta de manera considerable la base gravable del contribuyente y provoca un cálculo incorrecto del tributo a pagar.

La inexperiencia que pudieran tener los contribuyentes en temas tributarios, respecto a la deducibilidad de los bienes de la empresa, lo ha llevado a que busque formas de verse menos afectado a los cambios fiscales, tecnológicos, administrativos, etc.; lo cual redunda en un perjuicio.

Esto permite que existan Empresas Dedicadas a la Facturación de Operaciones Simuladas (EFOS) y por consecuencia que se deriven las Empresas que Deducen Operaciones Simuladas (EDOS). El SAT en los últimos años ha comprobado que las operaciones se han incrementado. 


\section{Figura 2.}

Aumento de EDOS y EFOS listado SAT

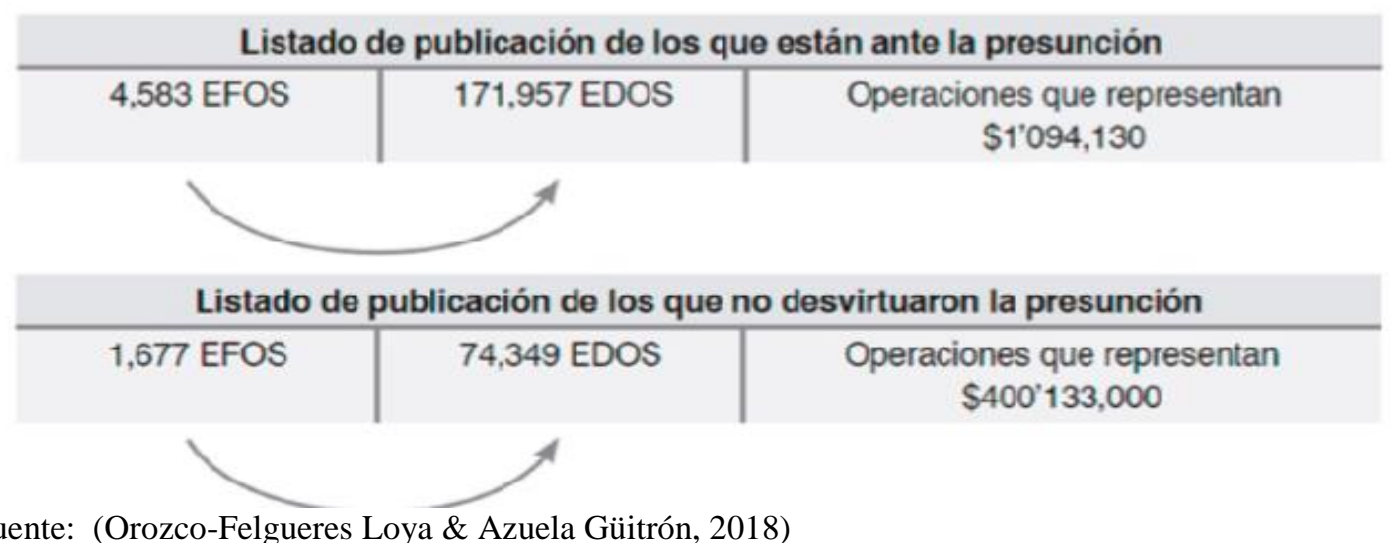

Fuente: (Orozco-Felgueres Loya \& Azuela Güitrón, 2018)

En el Diario Oficial de la Federación (DOF) publicado el 9 de diciembre de 2013, se reformaron artículos del CFF, que "tiene como finalidad atacar conductas y prácticas evasoras sancionando a quienes adquieren, venden, producen, facilitan y/o utilizan cualquier documentación que acredite una operación simulada”. (De Anda Turani, 2020).

El cambio realizado al Artículo 69-B del CFF señala:

Cuando la autoridad fiscal detecte que un contribuyente ha estado emitiendo comprobantes sin contar con los activos, personal, infraestructura o capacidad material, directa o indirectamente, para prestar los servicios o producir, comercializar o entregar los bienes que amparan tales comprobantes, o bien, que dichos contribuyentes se encuentren no localizados, se presumirá la inexistencia de las operaciones amparadas en tales comprobantes (Código Fiscal de la Federación, 2019).

El contribuyente será notificado mediante su buzón tributario en caso de encuadrar en el supuesto señalado, se le dará un plazo de 30 días, para que el contribuyente pueda manifestar lo que a su derecho convenga y presentar documentación para desvirtuar los hechos que llevo a la autoridad a notificar. A esta documentación que se adjunta en el buzón será la comprobatoria a la que se le llama materialidad de los hechos. 


\section{3 Materialidad}

Ya que la autoridad fiscal ha visualizado este acontecimiento, de la existencia de empresas que facturan y deducen operaciones comerciales o de servicio, toma en consideración que una manera de como poder corregir esas acciones es solicitar información adicional en la que el fisco se cerciore que realmente las transacciones se hagan con el debido respaldo, ejerciendo sus facultades de comprobación que señala el CFF y que si se encuentren cumpliendo con la legalidad.

Por el momento este concepto de materialidad, señala (Pérez, 2019) no se tiene contemplado en la legislación fiscal, lo cual puede generar consecuencias fiscales que perjudiquen al contribuyente, hasta que se obtengan criterios jurisprudenciales sólidos que arrojen certidumbre jurídica.

La Real Academia de la Lengua Española (RAE) define como la materialidad es un elemento que se integra de algunos compuestos que respaldan hechos centrales, en este caso a actividades administrativas, fiscales y financieros.

Partiendo de esta definición la materialidad, no considera un CFDI suficiente para respaldar acciones de compraventa o contratos elaborados entre ambas partes (comprador-vendedor), sino que es todo lo que sirva para comprobar que la acción mercantil se realizó de manera legítima y transparente.

Así la autoridad fiscal tiene como parte de su facultad de comprobación solicitar los documentos necesarios para constar que las acciones mercantiles sean verídicas entre ellos pide se muestren además estados de cuenta, CFDI, registros contables, papeles de trabajo, verificación mediante la observación directa, entre otros.

La Suprema Corte de Justicia de la Nación señala en materia de comprobantes fiscales, concepto, requisitos y funciones que:

Los comprobantes fiscales son los medios de convicción a través de los cuales los contribuyentes acreditan el tipo de actos o las actividades que realizan para efectos fiscales. Por su parte, El Código Fiscal de la Federación (2014, art. 29) en las fracciones I y VI, establece que los sistemas y registros contables deben identificar cada operación, acto o actividad y sus características, relacionándolas con la documentación 
comprobatoria. De tal forma que aquellos puedan identificarse con las distintas contribuciones, para acreditar la materialidad de las operaciones consignadas en ellos, ya que para que se considere real y probado su contenido, es indispensable que estén respaldados en los elementos documentales que comprueben que lo consignado en ellos, existió realmente; lo que implica la condición de contar con la documentación comprobatoria idónea para que se acredite el contenido de las operaciones detalladas. (IDC Online, 2013, párr. 1)

Ante esta circunstancia que se está viviendo se observa que hay un sinfín de datos que la autoridad fiscal puede solicitar al contribuyente para acreditar que realmente se están ejerciendo actos legítimos a las actividades comerciales, pero ¿Qué se debe hacer para acreditar las exigencias del fisco para sustanciar las actividades económicas del contribuyente?

Mientras esto sucede lo más recomendable es recabar la información y documentación posible que sirvan de prueba sobre las operaciones que realiza un ente económico, para así cumplir de manera cabal todas las formalidades legales que establece el fisco y lograr de esta manera una información sustancial.

\section{4 Certeza jurídica}

Es cierto que la certeza jurídica debe ofrecer confianza a los ciudadanos, es un principio que el estado debe conceder para obtener credibilidad en la materia en donde se desarrolle dicho acto.

Esto derivado de lo establecido en el Artículo 1 de la Constitución Política de los Estados Unidos Mexicanos (CPEUM), “Todas las autoridades, en el ámbito de sus competencias, tienen la obligación de promover, respetar, proteger y garantizar los derechos humanos de conformidad con los principios de universalidad, interdependencia, indivisibilidad y progresividad.” (Constitución Política de los Estados Unidos Mexicanos, 2021)

Por consiguiente, las autoridades hacendarias tienen la obligación de garantizar que los derechos humanos sean protegidos en la jurisdicción que les corresponda, atendiendo a los 
derechos fundamentales considerados en la CPEUM.

Por su parte, el criterio del poder judicial de la federación señala lo siguiente: “...en el caso de normatividad con efectos económicos o tributarios, por regla general, la intensidad del análisis constitucional debe ser poco estricta," (Análisis constitucional, su intensidad a la luz de los principios democrático y de división de poderes, 2006).

La manera de interpretación de las normas tributarias primordialmente es el método literal ya que se busca el sentido de los términos tributarios establecidos en ley, pero a falta de ésta se aplicará principios generales del derecho, como señala el segundo párrafo del CFF de su Artículo 5 establece que “.... Las otras disposiciones fiscales se interpretarán aplicando cualquier método de interpretación jurídica. A falta de norma fiscal expresa, se aplicarán supletoriamente las disposiciones del derecho federal común cuando su aplicación no sea contraria a la naturaleza propia del derecho fiscal." (Código Fiscal de la Federación, 2019).

Por lo tanto, se puede mencionar conforme al criterio normativo que los legisladores ejercen sobre las leyes tributarias como poco estrictas se vislumbra una diferencia muy clara entre lo mencionado al Artículo 1 CPEUM y lo establecido en jurisprudencia, por lo cual la certeza jurídica que los derechos humanos que el contribuyente tiene por el simple hecho de ser un ciudadano en territorio mexicano se ven violentados.

La realidad es que al no gozar de dicha certeza jurídica y de no haber ley que regule dicha revisión, la autoridad puede ejercer sus facultades de comprobación y desvirtuar la comprobación de gastos, perjudicando la base gravable, fundamentando que no cumplimos con lo que ellos consideran como materialidad.

Este hecho es el que centra el objeto de estudio que parte del hecho actual, vigente y que puede incluir a todo un régimen de tributación que realice operaciones comerciales donde resulten saldos a favor, con esto se demuestra la trascendencia del problema abordado.

\section{METODOLOGÍA, TÉCNICAS Y MATERIALES EMPLEADOS}

Esta investigación parte de un hecho específico que impacta a un $40 \%$ de los contribuyentes 
registrados; por ello se clasifican como una investigación cualitativa de acuerdo con (Bernal, 2010) que parte de las ciencias sociales al investigar el impacto en la sociedad.

Se fundamenta en la aplicación de la norma tributaria como técnica de indagación que utiliza como fuente documental primaria la cual se complementa con la observación directa e incluso participativa por la asesoría profesional que se brinda a las entidades económicas. El discurso empleado en este trabajo utiliza el método deductivo, llegando a la particularidad del caso en específico. Dentro de las técnicas y materiales utilizados se encuentran los casos que como el estudio se conocen de cerca por la asesoría que se brinda.

\section{DISCUSIÓN}

Derivado al estudio efectuado se tiene por resultado lo siguiente:

Que las autoridades hacendarias dentro de sus facultades de comprobación pueden exigir a los contribuyentes demostrar durante su revisión material de las operaciones, ya que se ha visto respaldado por los criterios jurisprudenciales y que estos tienen una tendencia al alza, ya que en las revisiones efectuadas por las autoridades hacendarias son pocos los momentos en que no solicitan la materialidad de los hechos (Martínez, 2019).

Mediante el Artículo 69-B CFF llega a existir la presunción de las actividades simuladas si la autoridad fiscal llega a detectar:

- Emisión de comprobantes fiscales sin contar con los activos, personal, infraestructura o capacidad material para poder comercializar los productos establecidos por el contribuyente.

- El no estar localizado.

Por lo cual el contribuyente tendrá la opción de poder desvirtuar los hechos notificados por parte del SAT, como lo establece la ficha de trámite 156/CFF con título "Informe y documentación que deberá contener la manifestación con la cual se desvirtué la presunción del artículo 69-B del código fiscal de la federación"

Puesto que no hay una manera establecida de que requisitos específicos para acreditar las transacciones, y no establecer parámetros bien definidos para la acreditación de los tratos comerciales, se deja al particular en un estado de indefensión. 
Por lo tanto, la certeza jurídica que pueden tener los elementos de materialidad con los que puede contar el contribuyente podrían o no ser suficientes para el fisco cuando efectúa sus facultades de comprobación.

Y al no contar con un criterio definido se hace más complejo el proceso administrativo que debe ejercer el contribuyente para desvirtuar los hechos de operaciones inexistentes, en lo cual se recomienda contar con toda la documentación posible para probar lo contrario y en caso de que sea necesario tramitar algún medio de defensa, esto puede ser incluso más costos para el sujeto tributario.

\section{CONCLUSIONES}

Legalmente, la manera para comprobar la materialidad de las transacciones se encuentra en los CFDI tanto emitidos como recibidos, no obstante, se complementan con los registros contables, contratos comerciales, cotizaciones, entre otros. La práctica que realiza la autoridad fiscal puede resultar correcta, asertiva y justificada cuando tiene información de que el contribuyente se encuentre falseando documentación, pero en otro momento se puede considerar de forma injustificada y llegar al punto de ser ilegal.

Puede ser correcto el procedimiento que aplica la autoridad para evitar la simulación de actos más la frecuencia con que la autoridad fiscal cuestiona la materialidad de las operaciones comerciales entre contribuyentes es altamente recurrente con un mismo contribuyente, llegando a originar la determinación de impuestos mayores al disminuir deducciones de la base gravable.

Las facultades de comprobación incluyen varias formas de realizar una verificación, entre ellas las compulsas y esa es una forma eficiente de demostrar que la operación se efectuó.

Al realizar esta indagación y los hechos analizados que han dado origen a la problemática se puede establecer otras interrogantes, ¿Bajo qué términos podemos saber que las pruebas presentadas son lo suficientemente congruentes para desvirtuar la presunción de las operaciones? ¿Quién decide si la evidencia presentada es suficiente? 
Es común observar la simple negativa del fisco federal a valorar ciertas pruebas que cumplen con todos los requisitos, haciendo que el contribuyente acuda a juicio para demostrar la existencia de sus operaciones.

Los órganos jurisdiccionales deben observar que la autoridad actué de manera razonable ante la facultad de comprobación al revisar los documentos del contribuyente. Y tener presente que la falta de certeza jurídica de un documento que ha sido autorizado por la misma autoridad es real, por ello deben dejar bien establecido un proceso para acreditar y deducir las actividades de la empresa y después no se conviertan en no verídicas, no transparentes y sobre todo congruentes.

Para finalizar es recomendable que el contribuyente prevea reunir todo lo que ocupó para realizar la transacción relacionada a su actividad económica como son contratos, CFDI, presupuestos, fotografías de los insumos que ocupo, correos electrónicos, teléfonos de proveedores, entre otros para poder demostrar a la autoridad fiscal que realmente se están ejerciendo de manera correcta las relaciones comerciales en la realización de actividades económicas y que está cumpliendo correctamente las obligaciones fiscales a las cuales se encuentra sujeto. En caso de que dicha autoridad fiscal mencione que no es suficiente los documentos señalados, entonces ejercer algún recurso de defensa que se establece en ley y CFF.

\section{REFERENCIAS}

Análisis constitucional, su intensidad a la luz de los principios democrático y de división de poderes, 1a./J. 84/2006 (Primera Sala de la Suprema Corte de Justicia de la Nación Noviembre de 2006).

Bernal, C. A. (2010). Metodología de investigación administración, economía, humanidades y ciencias sociales. Colombia: $\mathrm{P}$

Código de Comercio (2018). México.

Código Fiscal de la Federación. (2019).México.

Consejo Emisor del CINIF. (2005). Norma de Información Financiera A-3. Obtenido de Facultad de 
Contaduría y Administración : http://fcaenlinea1.unam.mx/anexos/1165/1165_u2_a3.pdf

Constitución Política de los Estados Unidos Mexicanos (Vigente). México.

Comisión Nacional del Sistema de Ahorro para el Retiro . (2018). Once desafíos para mejorar el SAR. Obtenido de Gob.mx: https://www.gob.mx/consar/articulos/once-desafios-para-mejorar-el-sar

CINIF. (2019). Normas de Información Financiera 2019. México: CCDM.

De Anda Turani, J. A. (14 de Mayo de 2020). Instituto Mexicano de Contadores Públicos IMCP. Obtenido de Instituto Mexicano de Contadores Públicos IMCP: https://imcp.org.mx/publicaciones/el-imcp-comunica-fiscalizacion-de-operaciones-simuladas-2/

Gurria, A. (2018). OCDE. Obtenido de http://www.oecd.org/mexico/oecd-review-pension-systemsmexico.htm

IDC Online. (22 de Julio de 2013). IDC Online. Obtenido de IDC Online: https://idconline.mx/fiscal/2013/07/22/requisitos-y-funciones-de-los-comprobantes-fiscales

Juicio Contencioso Administrativo, 582/17-29-01-6/875/18-S1-02-04. (Sala Superior del Tribunal Federal de Justicia Administrativa 7 de Agosto de 2018). Recuperado el 08 de Enero de 2020

Ley del Impuesto Sobre la Renta (2019). México.

Loya, C. O.-F. (2018). La materialidad de los actos jurídicos. Ciudad de México: Thomson Reuters .

Martínez, 2. (2019). Obligaciones de demostrar la materialización de las operaciones amparadas con comprobantes fiscale. Análisis jurídico. Práctica Fiscal, D1.

Organización para la Cooperación y el Desarrollo Económicos. (2016). Estudio de la OCDE sobre los sistemas de pensiones: México 2015. Obtenido de OCDE: http://www.oecd.org/daf/estudio-dela-ocde-sobre-los-sistemas-de-pensiones-mexico-2015-9789264250017-es.htm

Organización para la Cooperación y el Desarrollo Económicos. (30 de abril de 2019). OCDE México. Obtenido de OCDE: http://www.oecd.org/centrodemexico/

Pérez Martínez, V. M. (11 de Abril de 2019). IDC Online. Obtenido de IDC Online: https://idconline.mx/fiscal-contable/2019/04/01/materialidad-de-las-operaciones-para-efectosfiscales

Secretaría de Gobernación. (28 de 02 de 2019). SEGOB - Diario Oficial de la Federación. Obtenido de SEGOB - Diario Oficial de la Federación: 
https://www.dof.gob.mx/nota_detalle.php?codigo=5551425\&fecha=28/02/2019

Sistema de Administración Tributario. (30 de abril de 2019). SAT. Obtenido de SAT: https://www.sat.gob.mx/home

Tributaria, S. d. (13 de Mayo de 2020). Recuperado el 13 de Mayo de 2020, de SAT site: http://omawww.sat.gob.mx/cifras_sat/Paginas/datos/vinculo.html?page=giipPorRegimen.html 